CERN-PPE/92-29

17 February 1992

\title{
The gain monitoring system of the Aleph Hadron Calorimeter
}

\begin{abstract}
A. Messineo ${ }^{3}$, G. Raso ${ }^{1}$, A. Antonelli ${ }^{2}$, C. Avanzini ${ }^{3}$, G. Bagliesi ${ }^{3}$, G. Bencivenni ${ }^{2}$, F. Bossi ${ }^{2,4}$, U. Bottigli ${ }^{3}$, C. Bradaschia ${ }^{3}$, P. Campana ${ }^{2}$, G. Capon ${ }^{2}$, F. Cerutti ${ }^{2}$, V. Chiarella ${ }^{2}$, D. Creanza ${ }^{1}$, M. de Palma ${ }^{1}$, B. D’Ettorre-Piazzoli ${ }^{2,5}$, A. Farilla ${ }^{1}$, G. Felici ${ }^{2}$, L. Foà ${ }^{3}$, A. Giassi ${ }^{3}$, G. Iaselli ${ }^{1,4}$, P. Laurelli ${ }^{2}$, F. Ligabue ${ }^{3}$, G. Maggi ${ }^{1}$, M. Maggi ${ }^{1}$, E.B. Mannelli ${ }^{3}$, G. Mannocchi ${ }^{2,5}$, F. Murtas ${ }^{2}$, G.P. Murtas ${ }^{2}$, S. Natali ${ }^{1}$, S. Nuzzo ${ }^{1}$, F. Palla ${ }^{3}$, L. Passalacqua ${ }^{2}$, M. Pepe-Altarelli ${ }^{2}$, P. Picchi ${ }^{2,6}$, M. Quattromini ${ }^{1}$, A. Ranieri ${ }^{1}$, F. Romano ${ }^{1}$, F. Ruggieri ${ }^{1}$, G. Sanguinetti ${ }^{3}$, G. Selvaggi ${ }^{1}$, L. Silvestris ${ }^{1}$, P. Tempesta ${ }^{1}$, R. Tenchini ${ }^{3}$, J. Walsh ${ }^{3}$, G. Zito ${ }^{1}$
\end{abstract}

1. INFN Sezione di Bari e Dipartimento di Fisica dell' Università, 70126 Bari, Italy

2. Laboratori Nazionali dell'INFN (LNF-INFN), 00044 Frascati, Italy

3. Dipartimento di Fisica dell'Università, INFN Sezione di Pisa, e Scuola Normale Superiore, 56010 Pisa, Italy

4. Now at CERN

5. Istituto di Cosmo-Geofisica del C.N.R., Torino, Italy

6. Istituto di Fisica Generale, Università di Torino,Torino, Italy

\begin{abstract}
The gas gain monitoring system of the Aleph Hadron Calorimeter is described. The dependence of the charge response to a) the ratio between the pressure to temperature $b$ ) the gas mixture parameters ( $\mathrm{Ar} / \mathrm{CO}_{2}$ ratio and isobutane percentage) have been determined. The total gain variation is measured with a precision of about $0.4 \%$.
\end{abstract}

(Submitted to N.I.M.) 



\section{Introduction}

The Aleph Hadron Calorimeter (HCAL) [1] is an iron-plastic streamer tube[2] sampling calorimeter. It is divided in a central barrel and two endcaps and consists of 23 active layers interleaved in the iron of the magnet return yoke. Each active layer is equipped on one side with pad readout organised in projective towers for an integrated energy flux measurement and, on the other side, with strips, running parallel to the wires for a digital pattern read-out[3] of the events. The energy determination is based on the measurement of the total charge $\mathrm{Q}$ induced on the pads during the shower development. If $\mathrm{N}$ is the number of streamers produced by a crossing particle, the collected charge $\mathrm{Q}$ can be estimated using the relation:

$$
Q=N \cdot Q_{S}
$$

where $Q_{S}$ is the average single streamer charge. Assuming that small variations in the gas conditions do not affect the value of $\mathrm{N}$, the collected charge $\mathrm{Q}$ is affected only by changes in the average charge of single streamers. Therefore monitoring $Q_{S}$ is the key tool to achieve stable HCAL operation conditions.

The aim of the gain monitoring system is twofold:

- determine, periodically, the variation of the calibration constant relative to the nominal value.

- monitor the HCAL gas mixture quality.

This is accomplished by monitoring the single streamer charge distribution in a set of test tubes, the local atmospheric pressure and the room temperature. We have found that the dependence of $Q_{S}$ on the atmospheric pressure $(P)$, room temperature $(T)$ and gas mixture, can be expressed as:

$$
\frac{\Delta Q_{S}}{Q_{S}}=C_{\mathrm{x}} \frac{\Delta \mathrm{x}}{\mathbf{x}}+\delta(\text { gas })
$$

where $\mathbf{x}$ is the ratio between the atmospheric pressure and the absolute temperature $(\mathrm{x}=$ $P / T)$ and $C_{\mathrm{X}}$ is a constant. The first term in the previous equation accounts for changes in the gas density, while the second one, as will be explained below, accounts for changes in the gas mixture.

\section{Gas mixture}

Since the detector is located in an underground area and a large volume of gas is involved, we are forced to use for safety reasons a gas mixture containing a small quantity of hydrocarbons. We have chosen a ternary mixture of

$\begin{array}{rr}\text { Argon } & 22.5 \% \\ \mathrm{CO}_{2} & 47.5 \% \\ \text { sobutane } & 30.0 \%\end{array}$

with the percentages defined by mass flowmeters at a level of stability of $0.1 \%$. This mixture has a working knee for the streamer mode at about 4050 Volts as shown in Fig. 1.

This mixture was studied in dedicated test-beam runs with a prototype detector: the relationship between the calorimetric signal and the shower energy is linear up to about $40 \mathrm{GeV}$ 
and show a saturation of $5 \%$ at $45 \mathrm{GeV}$. The energy resolution in the linearity range can be parametrized as:

$$
\frac{\sigma(E)}{E}=\frac{85 \% \pm 1 \%}{\sqrt{ } E}
$$

and a m.i.p. (muon) traversing the full calorimeter depth produces the same signal as an hadronic shower induced by a $3.4 \mathrm{GeV}$ pion.

\section{The monitoring system}

The gas flows inside HCAL via a standard CERN distribution system, with a flow of about $500 \mathrm{l} / \mathrm{h}$ for a detector volume of $5 \cdot 10^{4}$ liters. A gas line per layer of tubes in each module (HCAL consists of 12 modules in the barrel and 6 sextants in each end-cap) is generally provided with the exception of the first 7 layers of the end-caps which, due to their small volume, are connected in series on a single line. This corresponds to 23 distribution lines in each module of the barrel and to 16 in each module of the end-caps. The sensitive volume connected to the different distribution lines varies from line to line especially in the barrel, where the number of tubes in each layer increases moving from the inner layer to the outer one. One distribution line in each module of the barrel and one in each sextant of the end-caps is monitored with the system described in this paper. In the endcaps the monitored distribution lines are connected always to the same tube layer, while in the barrel two different tube layers are used. In this way sensitive volumes of three different sizes are monitored:

$$
\begin{aligned}
& \text { Vol. } 1=\text { endcap } \rightarrow 92 \text { liters } \\
& \text { Vol. } 2=\text { barrel layer } 14 \rightarrow 110 \text { liters } \\
& \text { Vol. } 3=\text { barrel layer } 23 \rightarrow 130 \text { liters }
\end{aligned}
$$

This choice allows us a better understanding of the time evolution of the gas mixture changes as will be expained in the next section.

The HCAL gain monitoring system consists of 96 streamer tubes and 24 collimated $106 R u$ sources. These tubes have the same structure as those installed in the calorimeter, but a fixed length of $50 \mathrm{~cm}$. The high voltage is supplied with the same power supply used for the calorimeter ${ }^{1}$ The tubes are mechanically coupled two by two to form a "tube unit". A source is placed inside each pair of such "tube units" illuminating both of them (see Fig. 2). For each monitored gas line a "tube unit" is connected in series to the input (IN-tubes) and another one to the output (OUT-tubes).

The two tubes belonging to the same "tube unit" are used for different purposes: the farther from the $106 R u$ source is used for triggering, the nearer provides the unbiased distribution of the single streamer charge (Fig. 3a). A digital-OR is formed from the signals induced on a pad $\left(8 \times 8 \mathrm{~cm}^{2}\right)$ placed on the outer side of each triggering tube. This digital-OR then generates the gate to the $\mathrm{ADC}^{2}$ and the trigger to a dedicated Hadron Calorimeter Processor (HCP) which reads the ADC. For each trigger only two channels, out of 96, are expected to have signal: the triggering tube and its partner. The trigger rates of the different tubes have been equalized by acting on the collimators which enclose the sources and on the distance between the sources and the "tube unit".

\footnotetext{
${ }^{1}$ Caen HV power supply model 127

${ }^{2}$ A 96 channel Fastbus ADC Lecroy model $1885 \mathrm{~N}$
} 


\section{Monitoring algorithm}

The monitoring procedure is divided in two parts: the first is executed directly in the HCP, the second one on the On-Line VAX.

The HCP computes the truncated mean of the single streamer charge distribution for each tube in the following way. After the ADC read-out, the pedestal is subtracted and the "tube unit" with non zero signal is identified. For every $m$ events coming from the $i-t h$ tube, $l$ events having the lowest charge contents and $h$ events having the highest charge contents are rejected. The remaining events are used to compute the partial mean charge relative to $i-t h$ tube:

$$
q=\frac{1}{(m-l-h)} \sum_{j=l+1}^{m-h} q_{j} \text { where } q_{j}<q_{j+1}<q_{j+2}
$$

and its variance:

$$
V=\sigma_{q}^{2}=\frac{1}{(m-l-h)}\left[s^{2}+\frac{l}{m}\left(q-q_{l+1}\right)^{2}+\frac{h}{m}\left(q-q_{m-h}\right)^{2}\right]
$$

where

$$
\begin{gathered}
s^{2}=\left(q^{2}\right)-(q)^{2} \\
q^{2}=\frac{\sum_{j=l+1}^{m-h} q_{j}^{2}}{(m-l-h)} \\
q_{l+1}, q_{m-h} \text { are the "truncation points" }
\end{gathered}
$$

In our case the values $m=10, l=1$ and $h=4$ have been used. If the total number of events coming from the $i-t h$ tube is $N$, the mean single streamer charge for the $i-t h$ tube, $Q_{i}$, is obtained by combining the $N / m$ partial means $q$, weighted by their variance $V$ :

$$
Q_{i}=\begin{array}{ll}
\sum_{n=1}^{N / m} \frac{q_{n}}{V_{n}} \\
\sum_{n=1}^{N / m} \frac{1}{V_{n}}
\end{array}
$$

while its variance is given by:

$$
\Delta_{i}=\sigma_{Q_{i}}^{2}=\frac{1}{\sum_{n=1}^{N / m} V_{n}}
$$

Note however that out of $\mathrm{N}$ events only $\mathrm{n}=N(m-l-h) / m$ events are actually used to compute the $Q_{i}$ and its variance.

The HCP evaluates, for each monitor tube $i$ :

(a) the mean charge $Q_{i}$ and $\sigma_{Q_{2}}$

(b) the number $n_{i}$ of events used to compute the mean charge

(c) the $q_{j}$ spectrum 
An example of the spectrum obtained with the truncated mean procedure ( $q_{j}$ spectrum) is show in Fig. 3b. It is evident that the procedure does not change the peak position of the $Q_{S}$ distribution. Every thirty minutes a task running on the On-line VAX reads the values computed by the HCP, fits a gaussian to the $q_{j}$ spectrum for each monitoring tube $i$ and compares the fitted parameters with a standard set of central values and cuts in order to establish the reliability of the data. Changes in the mean single streamer charge are interpreted according to the following criteria:

1. changes in the gas density due to variation in the atmospheric pressure or gas temperature affect simultaneously the response of the IN-tubes and of the OUT-tubes independently on the gas volume considered. Moreover such changes have the same amplitude.

2. changes in the gas mixture affect firstly the IN-tubes and, after some delay, the OUT-tubes. This delay, due to gas mixture propagation, is determined by the gas volume considered.

To isolate the genuine gas mixture variation, correction for the local atmospheric pressure and room temperature is nedeed. Therefore also the monitoring of this local conditions is implemented.

\section{$5 \quad$ Performances}

The coefficient $C_{\mathrm{x}}$ of equation (2), which relates the mean single streamer charge variations to the pressure and temperature changes, is determined experimentally using the monitoring system in a situation where the gas mixture is stable $(\delta($ gas $)=0)$.

Fig. $4 \mathrm{a}$ and $4 \mathrm{~b}$ show, for the same time interval, the variation $\Delta \mathrm{x} / \mathrm{x}$ and $\Delta Q_{S} / Q_{S}$ (averaged on all IN and OUT tubes) versus time, while Fig. 5 shows the distribution of several measurements of the ratio

$$
R=\frac{\Delta Q_{S}}{\underset{\mathrm{x}}{\Delta \mathrm{x}}} \quad \text { where } \mathrm{x}=\frac{P(m b a r)}{T(\circ K)}
$$

when $\delta($ gas $)=0$. From the distribution of Fig. 5 one determines the average value of the coefficient $C_{\mathrm{x}}$.

$$
C_{\mathrm{x}}=-6.15 \pm 0.04
$$

If $C_{\mathrm{x}}$ is correctly determined the quantity

$$
D=\frac{\Delta Q_{S}}{Q_{S}}-C_{\mathrm{x}} \frac{\Delta \mathrm{x}}{\mathbf{x}}=\delta(\text { gas })
$$

must be constant and equal to zero when the gas mixture is stable. This is indeed the case, as show in Fig. 4c where $D$ is plotted for a time interval of 20 days. The width of its distribution, shown in Fig. 6, represents the sensitivity of the monitoring system. A gaussian fits gives:

$$
\sigma_{D}=0.40 \%
$$

The average single streamer dependence on the gas composition has been also investigate by assuming a the simple linear parametrization for $\delta($ gas $)$ : 


$$
\delta(\text { gas })=C_{\mathbf{r}} \frac{\Delta \mathbf{r}}{\mathbf{r}}+C_{\mathbf{I}} \frac{\Delta \mathbf{I}}{\mathbf{I}}
$$

where $\mathbf{r}$ is the $A r / C O_{2}$ relative fraction, $\mathbf{I}$ is the isobutane percentage and $C_{\mathbf{r}}$ and $C_{\mathbf{I}}$ are constants.

Eq. (9) can then be written as :

$$
D=\frac{\Delta Q_{S}}{Q_{S}}-C_{\mathrm{x}} \frac{\Delta \mathbf{x}}{\mathbf{x}}=C_{\mathbf{r}} \frac{\Delta \mathbf{r}}{\mathbf{r}}+C_{\mathbf{I}} \frac{\Delta \mathbf{I}}{\mathbf{I}}
$$

By measuring $D$ for several small, controlled variations of $\mathbf{r}$ or $\mathbf{I}$, the $C_{\mathbf{r}}$ and $C_{\mathbf{I}}$ constants have been found to be:

$$
\begin{aligned}
& C_{\mathbf{r}}=0.38 \pm 0.06 \\
& C_{\mathbf{I}}=-2.23 \pm 0.13
\end{aligned}
$$

Therefore the sensitivity of the system given in (10) allows to detect a $\mathbf{r}(\mathbf{I})$ variation of $3.1 \%$ $(0.5 \%)$ at $3 \sigma_{D}$ level.

The quantity $D$ defined by equation (9) can be evaluated using the IN-tubes $\left(D_{I N}\right)$ or the OUT-tubes $\left(D_{O U T}\right)$ indipendently. A step, due to a sudden change in the gas composition, is clearly visible in Fig. 7a, which shows the behavior of $D_{I N}$. Figs. 7b,c,d show the time evolution, during the same period, of the difference

$$
\text { Diff }=D_{I N}-D_{O U T}
$$

for the three detector volumes previously defined (see sect. 3 ).

The plots show that the effects due to changes in the gas mixture appear in the OUT-tubes after about 7, 11, and 13 days depending on the size of the detector volume connected to the gas distribution line. It is evident that the size of the effect is similar to the one observed on the IN-tubes, even if with a slower rise time. However Fig. $7 \mathrm{~b}$ shows that the step in $D_{O U T}$ is still rather sharp. This indicates that changes in the gas mixture propagate through the detector sensitive volume as a wave with a velocity determined by the flow rate.

The quantity $D_{I N}$ is continuosly checked for each gas line ; an alarm is sent if this variable exceeds a preset limiting value.

\section{Determination of the calibration correction factor}

The time evolution of $Q_{S}$ is the basic element for the determination of the calibration constant of HCAL during running time, relative to its value at the start. For each non triggering tube we evaluate the factor $K_{i}$ defined as :

$$
K_{i}=\frac{Q_{i}}{Q_{i}^{\circ}}
$$

where $Q_{i}^{\circ}$ is the nominal charge determined at the starting of the Aleph data taking.

From $K_{i}$ one determines $K_{I N}$ and $K_{O U T}$ averaging over the IN-tubes and on the OUT-tubes respectively.

The correction factor $\mathbf{K}$ is then defined as:

$$
\mathbf{K}=W_{I N} K_{I N}+W_{O U T} K_{O U T}
$$


If no change in the gas mixture has been detected the two weights, $W_{I N}$ and $W_{O U T}$, are equal and both are set to 0.5. If, however, a gas change has been detected on the IN-tubes then the weights are assumed to be time dependent according to the plot shown in Fig. 8 . In fact gas mixture waves move through the detector with a velocity which depends on the flow rate and we can assume that the volumes in front and behind the wave are sharply separated. At a given time, a fraction of the detector will behave as the IN-tube and the other one as the OUT-tube depending on the position reached by the wave front of the gas mixture change. The use of time dependent weighting factors related to the fractions of the detector filled with the IN-like and OUT-like mixture allows a better estimate of the average behavior of the full calorimeter in the transition period.

\section{Conclusions}

A set of streamer tubes illuminated by collimated $106 R u$ sources is continously operating to monitor the gain of the Aleph Hadron Calorimeter.

An algorithm based on the truncated mean of the single streamer charge distribution allows to measure gain variations with a sensitivity of about $0.4 \%$.

The dependence of the charge response on the external pressure and temperature ratio $\mathrm{x}$ has been determined as well as the dependence on the gas mixture parameters $\mathbf{r}$ and $\mathbf{I}$ obtaining $C_{\mathrm{x}}=-6.15 \pm 0.04, C_{\mathrm{r}}=0.38 \pm 0.06$ and $C_{\mathrm{I}}=-2.23 \pm 0.13$.

After correction for effects due to the external conditions any change in the gas mixture is monitored with high sensitivity. By comparing the charge response of the IN-tubes to that of the OUT-tubes a time-dependent correction factor can be obtained which takes into account the finite velocity of the gas mixture discontinuity throughout the detector.

\section{Acknowledgements}

We take this opportunity to thank those who contributed to the construction and assembly of the ALEPH hadron calorimeter. In particular we would like to thank F. Ceglie, F. Chiumarulo, A. Clemente, R. Ferorelli, V. Negro, M. Papagni and C. Pinto of INFN Sezione di Bari; M. Anelli, A. Balla, M. Carletti, G. Corradi and M. Santoni of Laboratori Nazionali di Frascati; A. Bechini, M. Favati and G. Pagani of INFN Sezione di Pisa.

\section{References}

[1] D. Decamp et al., ALEPH Collab., Nucl. Instr. and Meth. A294(1990)121

[2] E. Iarocci, Nucl. Instr. and Meth., 217(1983)30

[3] M. G. Catanesi et al., Nucl. Instr. and Meth., A297(1990)390 


\section{Figure captions}

Figure 1 : The plateau curve of high tension for a monitor tube .

Figure 2 : The HCAL gain monitoring system layout.

Figure 3 : The single streamer charge distribution; a) full spectrum and b) trunctated spectrum.

Figure $4:$ The time behavior of a) $\left.\Delta Q_{S} / Q_{S}, \mathrm{~b}\right) \Delta \mathrm{x} / \mathrm{x}$ and c) $D=\frac{\Delta Q_{S}}{Q_{S}}-C_{\mathrm{x}} \frac{\Delta \mathrm{x}}{\mathrm{x}}$.

Figure 5 : The distribution of the ratio

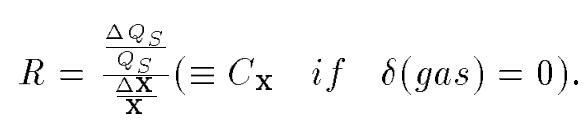

Figure 6 : The distribution of $D$ when $\delta($ gas $)=0$.

Figure 7 : The time behavior when $\delta($ gas $) \neq 0$ of a) $D_{I N}$, b) Dif $f($ vol.1), c) Diff(vol.2) and d) Diff(vol.3).

Figure 8: $W_{I N}$ and $W_{O U T}$ versus time when $\delta(g a s) \neq 0$. 


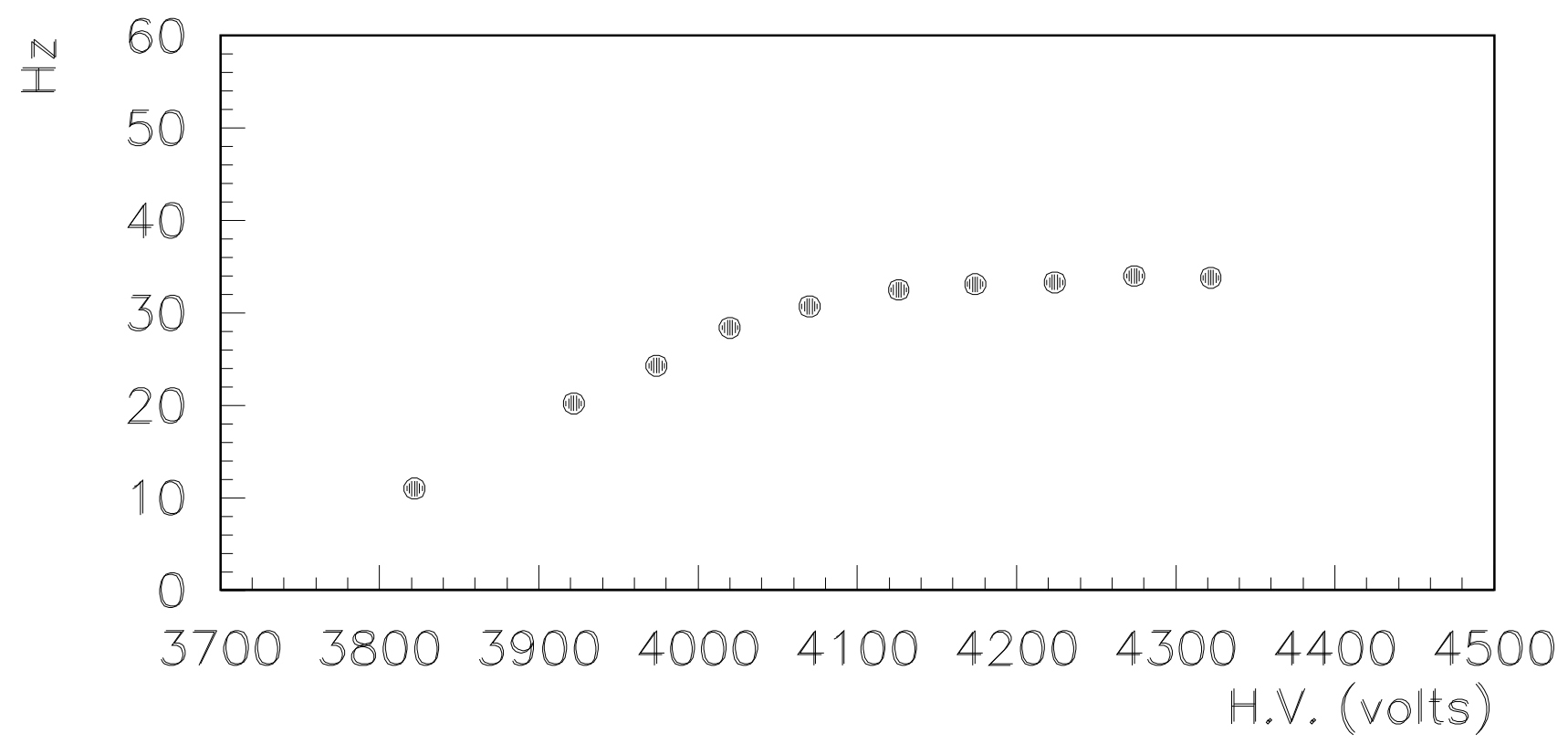

Figure 1: 


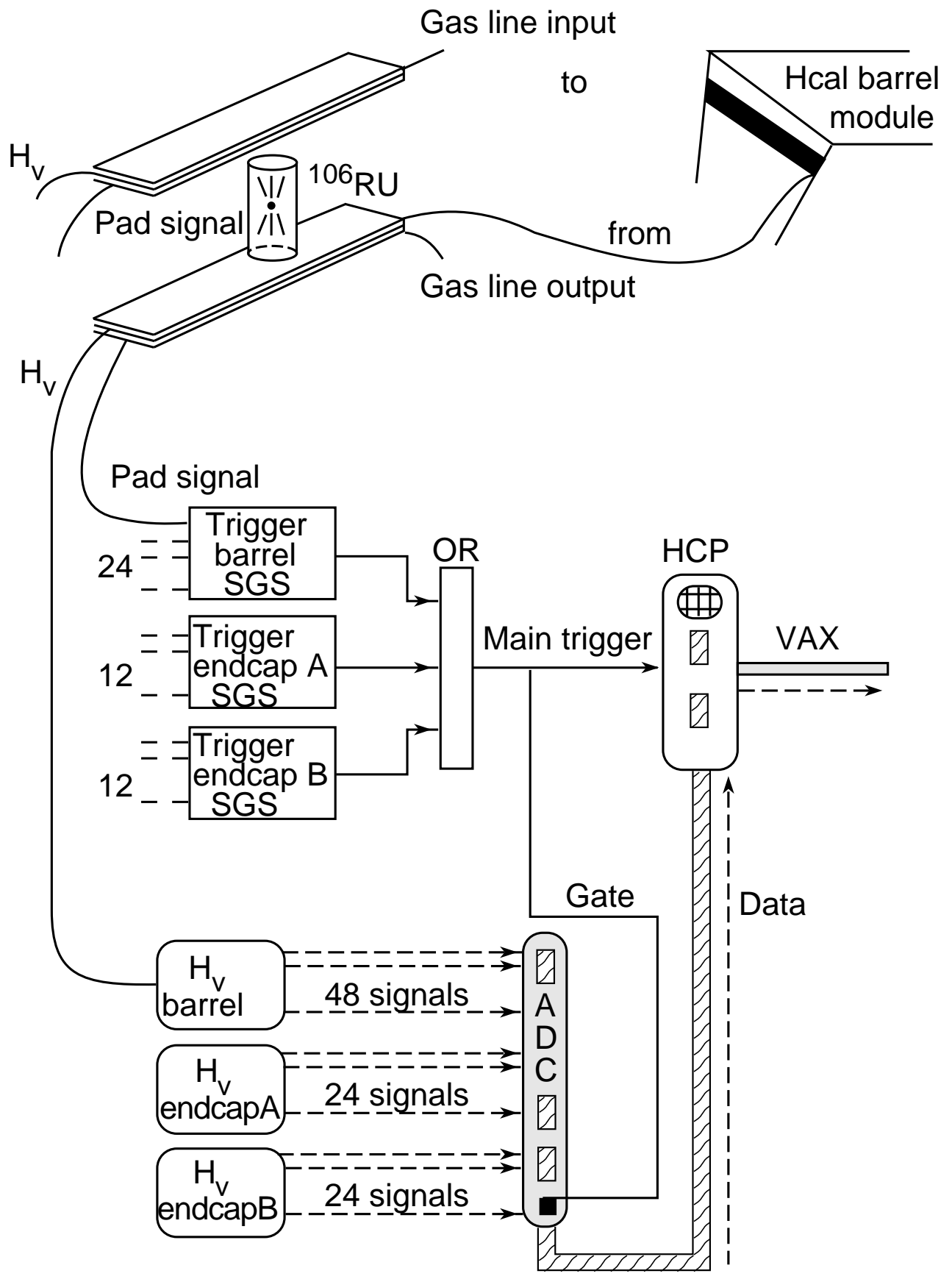

Figure 2: 

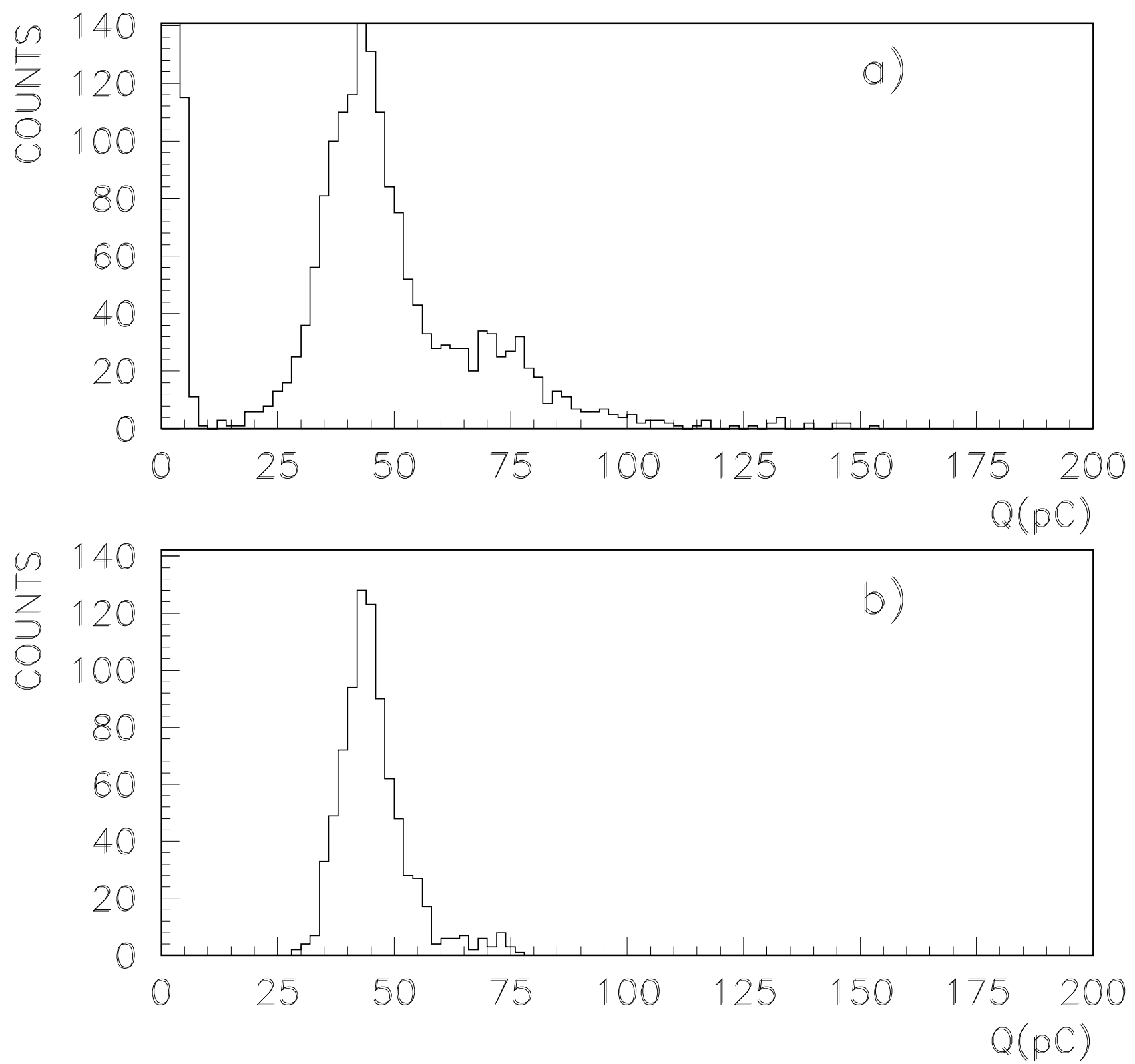

Figure 3: 

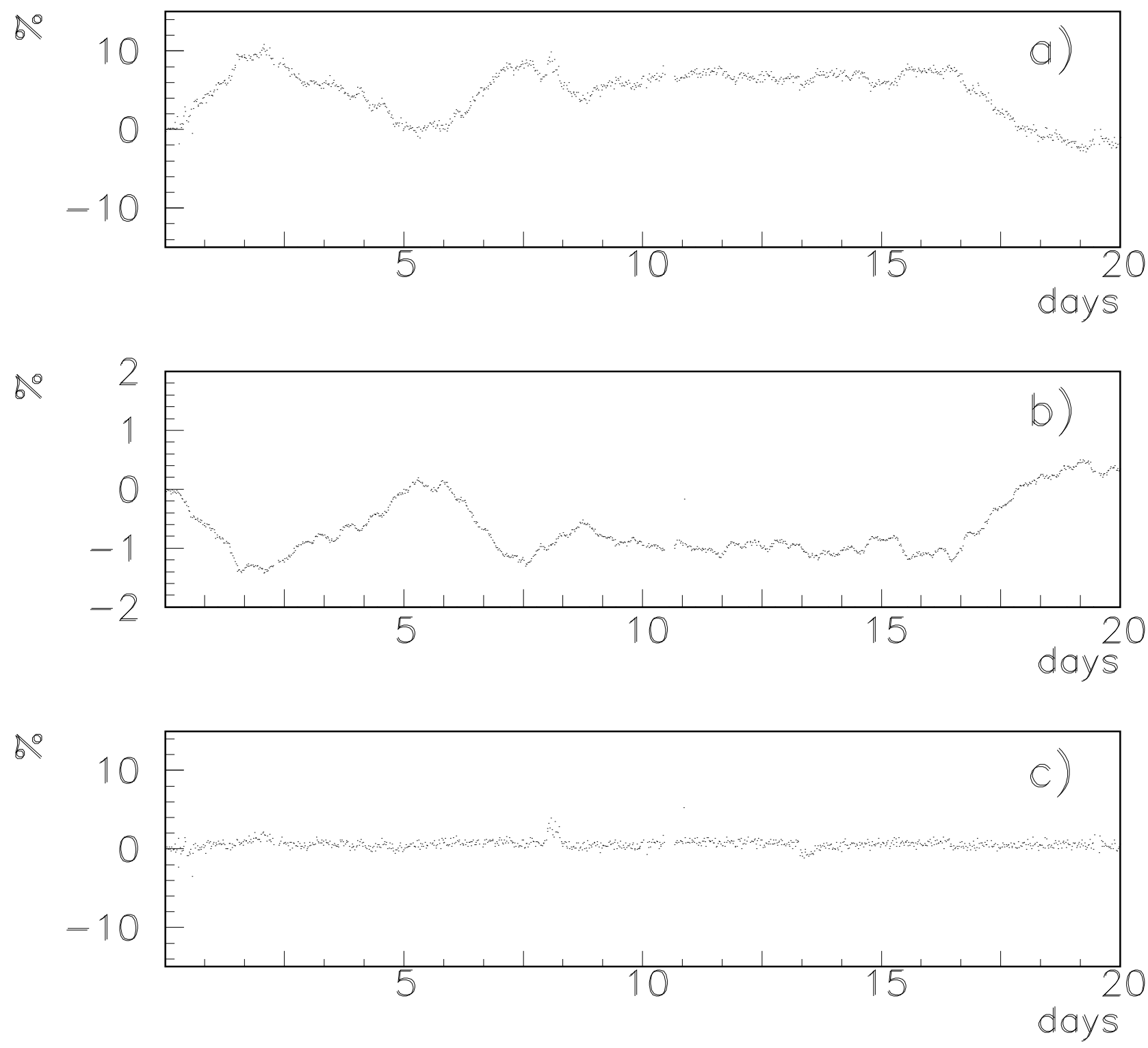

Figure 4: 


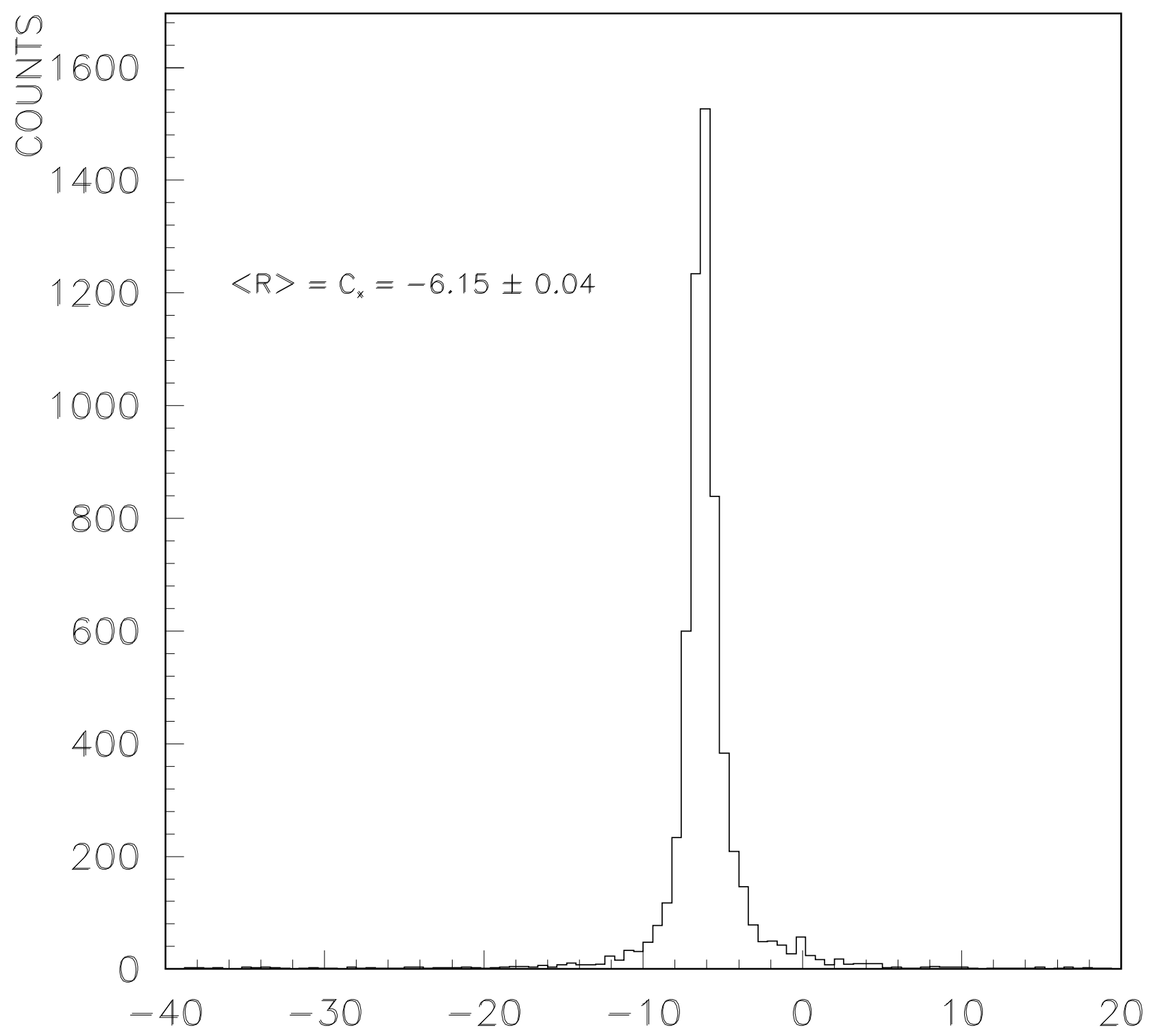

Figure 5: 


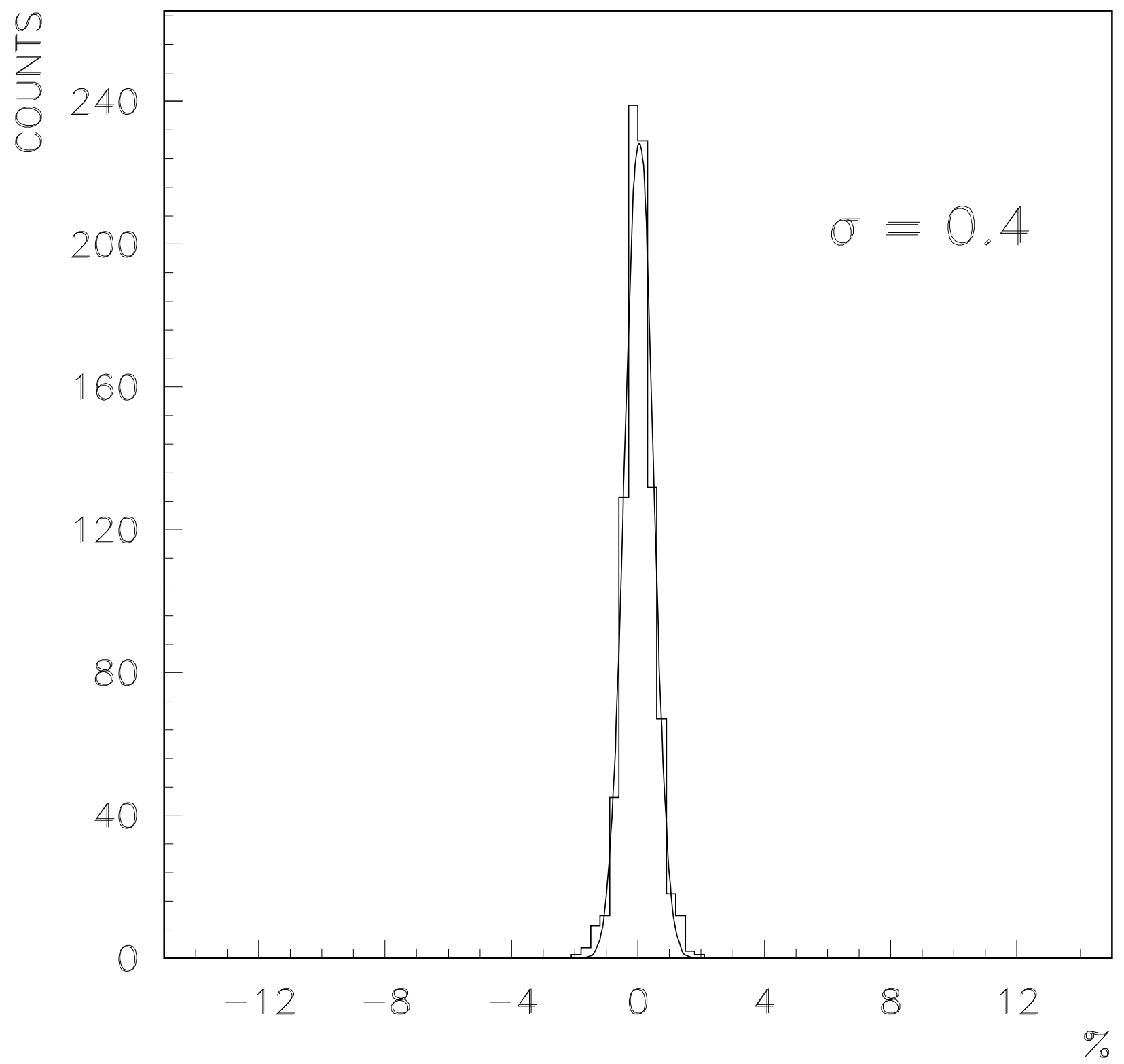

Figure 6: 

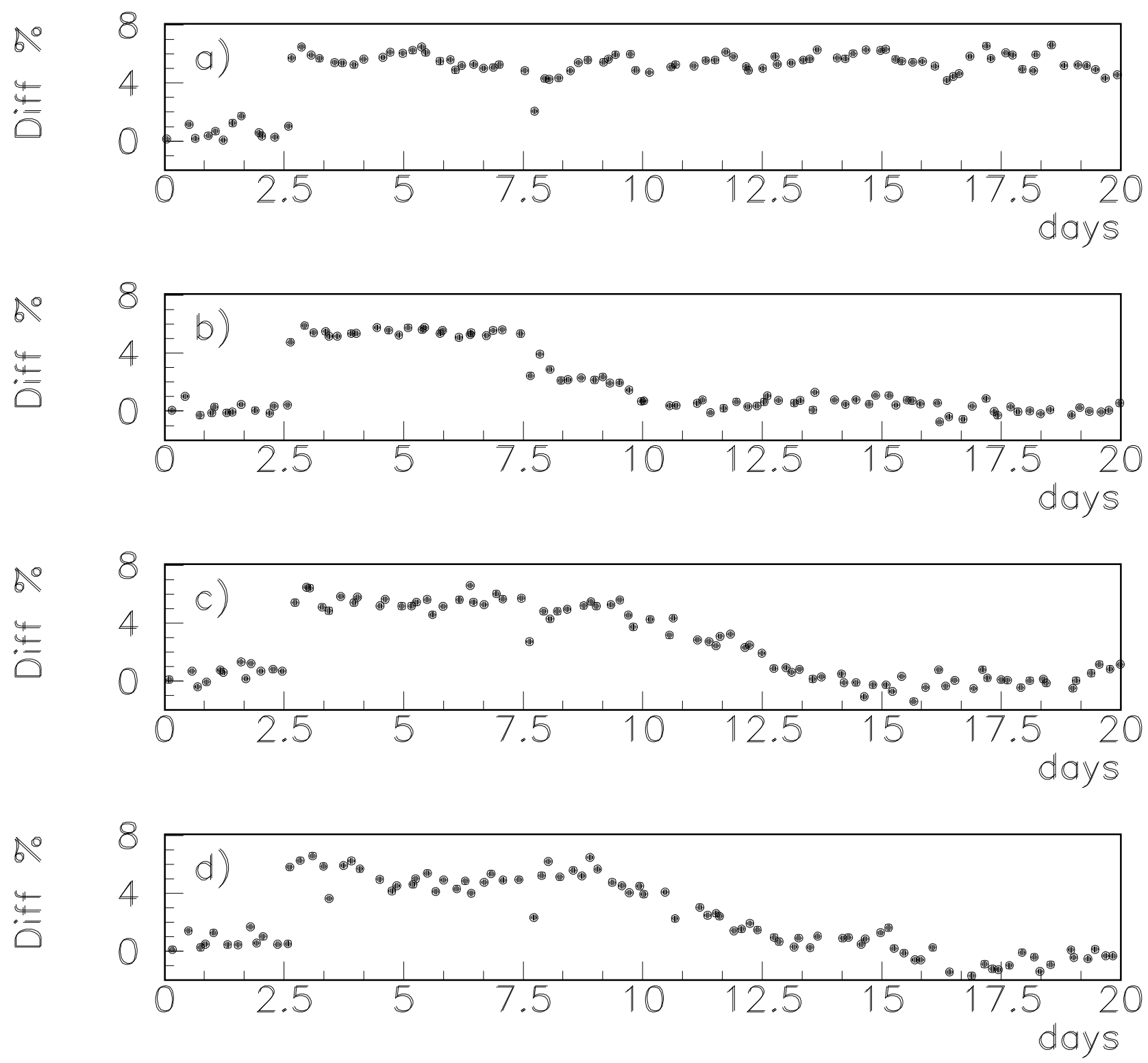

Figure 7: 


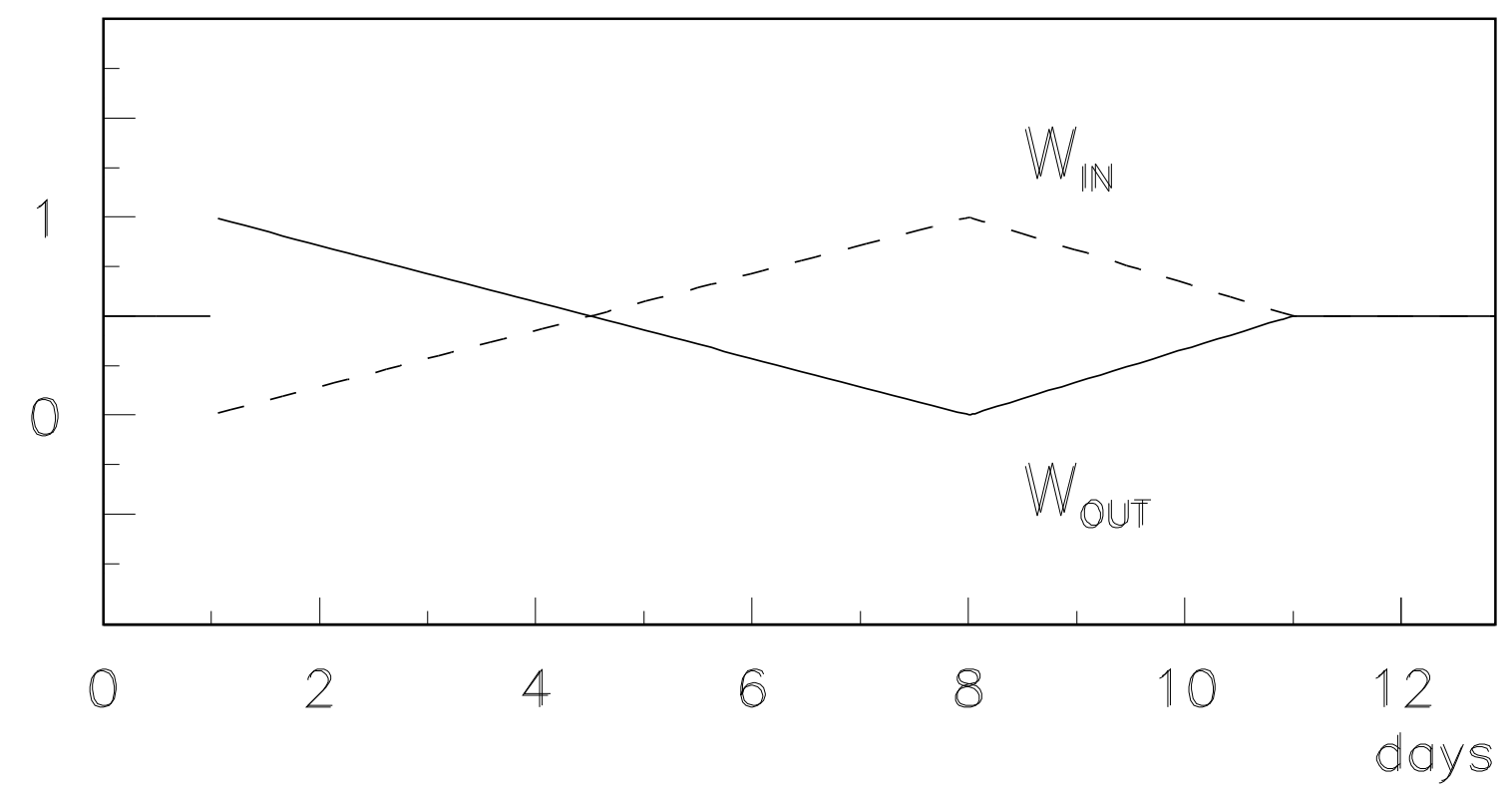

Figure 8: 